\title{
AUTOMATED TREATMENT OF FILM TEAR IN DEGRADED ARCHIVED MEDIA
}

\author{
D. Corrigan ${ }^{\dagger}$ and A. Kokaram \\ Department of Electrical and Electronic Engineering, \\ Trinity College Dublin, \\ Ireland.
}

\begin{abstract}
A common form of degradation in archived film is film tear. This is caused by the physical ripping of the film. Tear causes displacement of a region of the degraded frame and the loss of image data. As of yet no method of automatically treating film tear has been proposed. This paper outlines an algorithm to automatically detect and restore torn frames. Tear Detection is facilitated by the presence of a large edge feature, unlikely to be caused by other forms of degradation, in the the tom frame. Restoration is achieved by estimating the regional displacement and recovering missing image data.
\end{abstract}

\section{INTRODUCTION}

Recent years have seen the availability of digital media increase dramatically. The extra broadcast channels available have increased the demand for archived film and video. However the quality of archived material often does not match the high standards of quality demanded by digital media due to degradation of the film. The most common forms of degradation include intensity fluctuations in the film (known as flicker [1]) and presence of dirt and sparkle (also called blotches [2]) on the film. Another common form of film degradation is film tear which is caused by the physical ripping of the film. Like blotches, tear results in missing image data but additionally causes displacement in a region of the frame. Figure 1 indicates the physical displacement and the large edge feature caused by the tear. Up the present time tear was treated by manually highlighting the displaced region and then copying and pasting the region until the edges were properly aligned. This paper outlines a method to automate this process. The tear is diagnosed by the detecting the edge feature and then using motion information [3] and edge feature to delineate the tear. Treatment is performed by estimating the global motion separately for the regions either side of the tear and deciding which global motion corresponds to tear.

\footnotetext{
* This work was funded by a grant from IRCSET Ireland.

temail: corrigad@tcd.ie

†email: akokaram@tcd.ie
}

\section{TEAR DIAGNOSIS}

Tear Diagnosis is broken into two separate problems of detecting torn frames and delineating the tear within a detected frame (marking the location of the tear on the frame). This from our experience increases the speed of diagnosis by performing a quick high level scan to detect torn frames and then performing the intensive delineation processes on suspected frames only.

Torn frames are detected by looking for the presence of a large edge feature in a frame. Large Edge features are characterised by high gradient magnitudes and are found by calculating the skew, $S$, of a histogram of gradient magnitudes for each frame where the skew for frame $n, S_{n}$ is given by

$$
S_{n}=\frac{G_{n}^{99}}{\mu_{n}^{g}}
$$

where $\mu_{n}^{g}$ is the mean gradient magnitude and $G_{n}^{99}$ is the gradient magnitude bounding the top $1 \%$ of area of the histogram. A torn frame will result in a Skew measurement that is higher than that for the surrounding frames (See Fig 5).

Torn frames are isolated by imposing a gaussian model on the skew measurements and selecting measurements three standard deviations above the mean. This is then repeated omitting previously detected frames. Shifts in the mean caused by shot cuts are eliminated by subtracting the output of a five tap median filter from the skew measurements. Figure 5 shows the measurements for the two corrupted sequences. In order to test the accuracy of the detector a sequence of 20 frames was artificially corrupted with 5 tears. Due to the large concentration of tears in the sequence not all tears were detected even after the second pass despite the presence of well defined peaks in the skew measurements.

\subsection{Tear Delineation}

The problem is to construct a binary field, $b$, where

$$
b(\mathbf{x})= \begin{cases}1 & \mathbf{x} \text { is a tear site } \\ 0 & \text { otherwise }\end{cases}
$$


The field $b$ is constructed by using the MAP estimate of $b(\mathbf{x})$ that maximises the distribution $\left.P(b(\mathbf{x})) \mid G, \Delta_{f}, \Delta_{b}, B\right)$ where $G$ is a field of Gradient magnitudes for the frame, where $B$ donates the local neighbourhood of $b(\mathbf{x})$ and where $\Delta_{f}$ and $\Delta_{b}$ are the forward and backward Displaced Frame Differentials (DFDs) respectively. The DFDs measure the error in the motion estimation process in the forward and backward directions. According to Bayes Law the posterior can be reduced to

$$
\begin{aligned}
\left.P(b(\mathbf{x})) \mid G, \Delta_{f}, \Delta_{b}, B\right) \propto & P_{l}\left(G, \Delta_{f}, \Delta_{b} \mid b(\mathbf{x}), B\right) \\
& \times P_{s}(b(\mathbf{x}) \mid B)
\end{aligned}
$$

where $P_{l}$ is the data likelihood and $P_{s}$ is a spatial prior which applies a spatial smoothness constraint.

The Data Likelihood introduces the constraint that at tear sites the forward and backward DFDs and the gradient magnitude are large. It constraints the signs of DFDs to be the same. The expression used to calculate the likelihood, $P_{l}\left(G, \Delta_{f}, \Delta_{l} \mid b, B\right)$, is

$$
\begin{aligned}
& P_{l}\left(\Delta_{f}(\mathbf{x}), \Delta_{b}(\mathbf{x}), G(\mathbf{x}) \mid b(\mathbf{x}), B\right) \propto \\
& \exp \left\{-(1-b(\mathbf{x}))\left[\Lambda_{0}\left(\frac{\Delta_{f}(\mathbf{x})^{2}}{2 \sigma_{f}^{2}}+\frac{\Delta_{b}(\mathbf{x})^{2}}{2 \sigma_{b}^{2}}+\frac{G(\mathbf{x})^{2}}{2 \sigma_{g}^{2}}\right)\right.\right. \\
& \left.\left.+\Lambda_{s}\left(1+\operatorname{sign}\left(\Delta_{f}(\mathbf{x}) \Delta_{b}(\mathbf{x})\right)\right)\right]-\alpha b(\mathbf{x})\right\}
\end{aligned}
$$

where the DFDs, $\Delta_{b}$ and $\Delta_{f}$, and the gradient, $G$, are normalised by their variances, $\sigma_{b}, \sigma_{f}$ and $\sigma_{g}$ respectively; where $\Lambda_{0}$ and $\Lambda_{s}$ are equation parameters and where $\alpha$ is the energy for $b(\mathbf{x})=1$. In this case $\alpha$ is a constant and effectively acts as a threshold or as an energy penalty for $b(\mathbf{x})=1$.

The Prior $P_{s}(b \mid B)$ encourages spatial smoothness in the result. A Gibbs Energy Prior is adopted and is given by the following expression

$$
P_{s}(b(\mathbf{x}) \mid B) \propto \exp \left(-\Lambda_{b} \sum_{\mathbf{u} \in S_{N}(\mathbf{x})}|b(\mathbf{x})-b(\mathbf{u})|\right)
$$

where $S_{N}(\mathbf{x})$ is the 8 connected neighbourhood of $\mathbf{x}$. A value of 1 is used for the parameter $\Lambda_{b}$.

The Optimised Solution is obtained using the Iterated Conditional Modes algorithm [4]. The state of each pixel, $\mathbf{x}$, is given by the value of $b(\mathbf{x}), b(\mathbf{x})=0$ or $b(\mathbf{x})=1$, that maximises the posterior $\left.P(b(\mathrm{x})) ! G, \Delta_{f}, \Delta_{b}, B\right)$. Iteration is continued until there is no change in successive estimates. Typically convergence is exponential and occurs within ten iterations. The process is initialised by using a blotch detector to generate an estimate of the result. The Blotch detector used is based on the SDI detector $[2,5]$. The motion of objects is tracked forwards and backwards through a sequence using motion estimation techniques $[2,3]$. Since a tear is not present on consecutive frames it will not be matched by the estimator and will result in large forward and backward DFDs, $\Delta_{f}$ and $\Delta_{b}$, at tear sites and the sign of the DFDs will be the same. The SDI detector is defined by the expression

$$
B(\mathbf{x})= \begin{cases}1 & \left|\Delta_{f}\right|>\delta_{l},\left|\Delta_{b}\right|>\delta_{t}, \Delta_{b} \times \Delta_{f}>0 \\ 0 & \text { otherwise }\end{cases}
$$

where $B(\mathbf{x})=1$ implies that the pixel $\mathbf{x}$ is a tear site and $\delta_{t}$ is a threshold applied to the DFDs. Figure 6 shows the results for the SDI detector and the final outcome.

\section{TEAR TREATMENT}

Having delineated the tear the torn frame can be restored. Tear causes displacement in a region of the tom frame. In order to estimate the displacement it is necessary to calculate the global motion of the region. Figure 3 shows the vector field for two consecutive frames where the second image is of a torn framc. The distribution of the field on the torn frame differs significantly on either side of the tear and the magnitude of the vectors in the displaced bottom region are significantly larger. The displacement is calculated by dividing the frame into two regions either side of the tear and by calculating the modes of vector histograms of both regions (Figure 4 shows a contour map of the histograms for the two regions of a torn frame). The mode is an estimate of the global motion of the region. One of the two regions must be selected as the displaced region. Two observations can be made. The first is that displacement caused by tear is likely to be larger than displacement caused by other factors. The second is that motion of the displaced region is more coherent and hence the vectors are more concentrated about the mode of the histogram (See Figure 3). The ratio of histogram entropy to motion vector mean is used as it factors both observations in the decision. The region with the lower ratio is chosen.

A bin size of $1 / 8 \mathrm{pel}$ is used for the vector histograms. Vectors are also weighted according to their accuracy. The expression for the weight of a vector at pixel $\mathbf{x}, w(\mathbf{x})$ is given by

$w(\mathbf{x})=\mu(\|\mathbf{G}(\mathbf{x})\|) \times\left|\cos \left(\angle(\mathbf{G}(\mathbf{x}))-\angle\left(\mathbf{d}_{\mathbf{n}, \mathbf{n}-\mathbf{1}}(\mathbf{x})\right)\right)\right|$

where $G(x)$ is the gradient vector at $x$ and $d_{n, n-1}(x)$ is the motion vector at $\mathbf{x} . \mu(\|\dot{\mathbf{G}}(\mathbf{x})\|)$ is an accuracy index based on the local gradient magnitude and ranges from 0 for low gradient magnitudes to 1 for high magnitudes. This reults in large weights where the gradient magnitude is large and where the gradient vector and motion vector have similar phases. 
The restored image, $I_{r}$, is calculated using the following expression

$$
I_{r}(\mathbf{x})=r^{\prime}(\mathbf{x}) I_{d}(\mathbf{x})+r(\mathbf{x}) I_{d}\left(\mathbf{x}+\mathbf{D}_{\mathrm{t}}\right)
$$

where $\mathbf{D}_{\mathbf{t}}$ is the calculated regional displacement; where $I_{d}$ is the corrupted frame, where $r(\mathbf{x})$ is a binary field where a value of 1 indicates that the pixel $x$ is in the displaced region of the frame and where $r^{\prime}(\mathbf{x})$ is the compliment of $r(\mathbf{x})$. Bilinear interpolation is used to retrieve image data from non-integer pixel sites.

Figures 2 and 7 shows a close up of a frame which has been restored. The JOMBANDI $[2,6]$ missing data treatment process was used to recover missing data (caused by the destruction of film material) from the frame. See Figure 7. As no salisfactory method of dividing the frame into two regions was developed a picture editor was used to do this manually.

\section{FINAL COMMENTS}

This paper introduces an original method of automatically treating film tear. The use of histograms of motion vectors is crucial as it allows the displacement caused by tear to be estimated and removed. The displacement is accurately estimated as can be seen by the alignment of edges across the tear. The JOMBANDI process removes the edge feature from the frame and fully remove the effects of the tear. The Diagnosis algorithm accurately detects torn frames and the accuracy of the process will improve with longer sequences as the relative frequency of occurrence of tom frames decreases. Additionally this high level content retrieval is a useful aid to manual tear treatment by quickly finding torn frames. Improving the results for delineation and the treatment of rotational displacement are the the main areas of further study.

\section{REFERENCES}

[1] A. Kokaram, R. Dahyot, F. Pitie, and H. Denman, "Simultaneous luminance and position stabilisation for film and video," in Visual Communications and Image Processing, San Jose, Califomia, USA, 2003.

[2] Anil Kokaram, Motion Picture Restoration: Digital Algorithms for Artefact Suppression in Degraded Motion Picture Film and Video, Springer Verlag, 1998.

[3] J. Biemnond, L. Looijenga, D. E. Boekee, and R.H.J.M. Plompen, "A pel-recursive wiener based displacement estimation algorithm," Signal Processing, vol. 13, pp. $399-412,1987$.

[4] J.E. Besag, "On the statistical analysis of dirty pictures," Journal of the Royal Statistical Society B, vol. 48, pp. 259-302, June 1986.
[5] R. Storey, "Electronic detection and concealment of film dirt," SMPTE Journal, pp. 642-647, June 1985.

[6] Anil Kokaram and Simon Godsill, "Mcmc for joint noise reduction and missing data treatment in degraded video," IEEE Transactions on Image Processing, vol. 50, pp. 189-205, Februaury 2002.

[7] J. O'Ruanaidh and W. Fitzgerald, Numerical Bayesian Methods Applied to Signal Processing, Springer Verlag, New York, 1996.

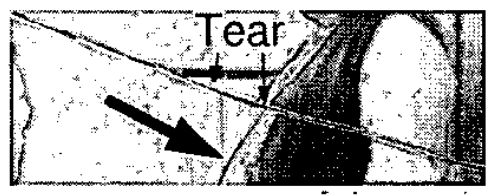

Fig. 1. An example of a torn frame. The arrows in the image indicate the regional displacement caused by the tear.

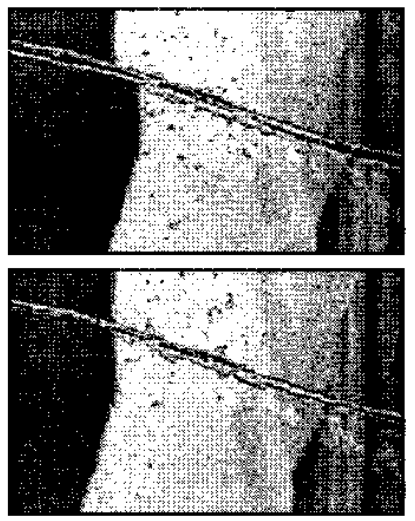

Fig. 2. Top image is a zoom in of the tom frame; The Bottom is a zoom of the treated frame.

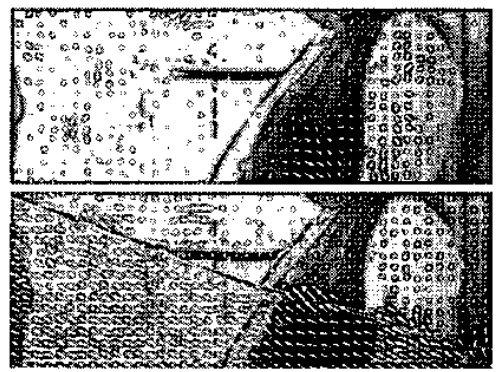

Fig. 3. Motion vector fields for two consecutive frames. The second image shows the torn frame. The vectors indicate the relative position of the previous frame to the current frame. 

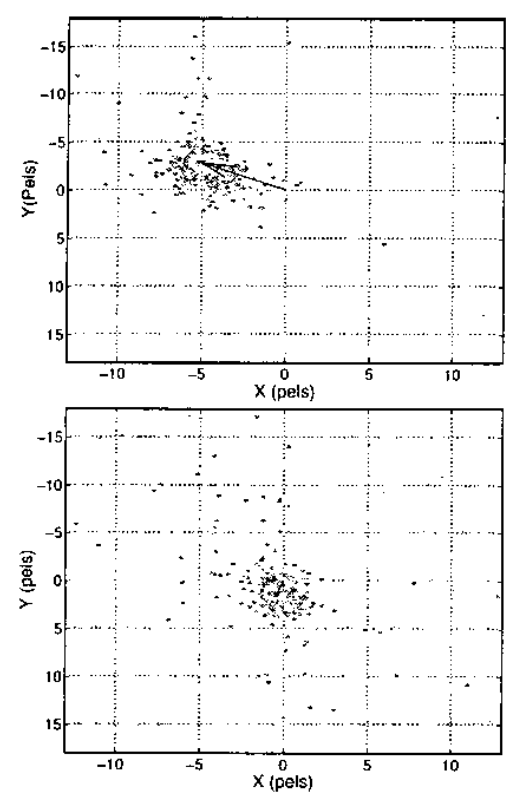

Fig. 4. Contour maps of the vector histograms for the two regions of the tom frame. The arrow points from the origin to the mode of the histogram.
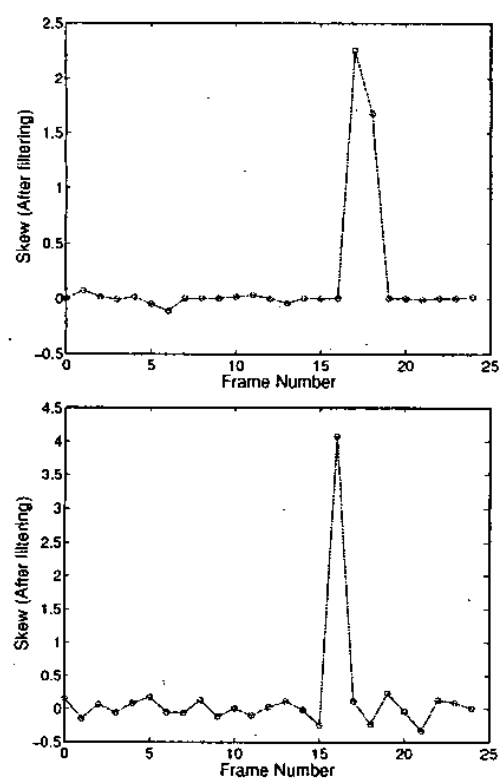

Fig. 5. Graphs show a plot of the skew values against frame numbers for two separate test sequences. There are 2 tears on consecutive frames in the first sequence and a single tear in the second.

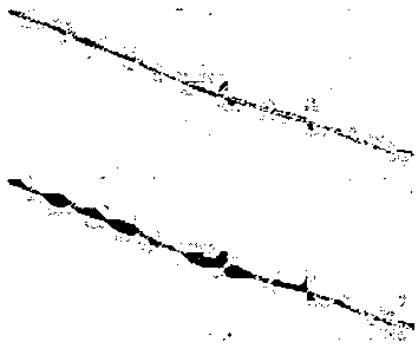

Fig. 6. Results of the Tear Delineation Process on the frame shown in Figure 1. The top map shows the results after the SDI detector and the bottom shows final result of the delincation process.
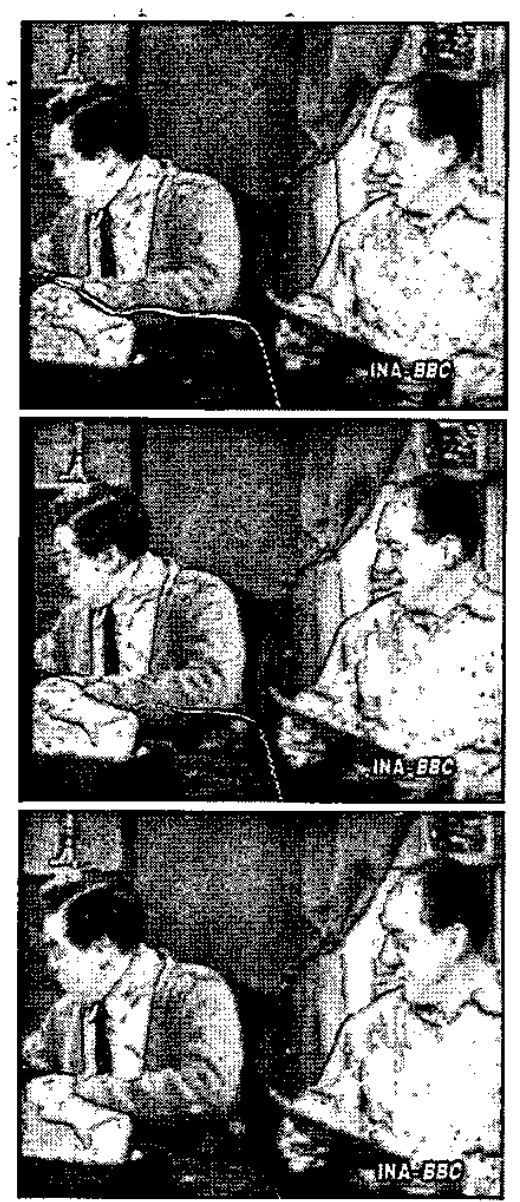

Fig. 7. Top image is the tom frame. The second is the image with tear displacement removed. The $3^{r d}$ is the fully restored frame after missing data treatment. Footage provided by INA-BBC as part of the AURORA, BRAVA and PRESTOSPACE projects. 